\title{
viewpoint
}

\section{Cell 'guidling'}

\author{
A binary logic to explain cell genesis \\ GuidoW.M.Shart
}

$\mathrm{L}$ ife is essentially information processing: signals from the environment are - transferred by signalling networks to the genome, where they are further processed to start or stop only those cellular functions that give the appropriate response. The mission of the life sciences in the genomics era is to decipher these instructions and thereby unravel the logic behind this information processing and how it translates to the behaviour and functions of living cells, organs and whole organisms. This article is an attempt to develop a new approach to this challenging task by using binary logic to explain how environmental signals are converted into cellular actions. An obvious starting point is a single cell, because all multicellular organisms originate from a single cell. A part from being an exercise in logic, this approach may help scientists to gain new insights and find new ways to tackle diseases that are caused by corrupted information processing, most notably cancer.

There are three basic rules for applying binary logic to living entities (H ofstadter, 1979) and for how the environment influences a binary founder cell's genicsmeaning all inherited abilities to react to environmental signals and conditions. The first rule states that the binary cell and its offspring are convinced of their autonomy. This implies that cells 'believe' they are free and independent to express their intrinsic genics, that they are not aware of any restrictions imposed on them by the environment, and that they ignore their past development and future potential. The second rule states that, unfortunately for the 'autonomous' cell, its environment imposes all kinds of restrictions and limits on the ability of the cell to express its genics. Stunningly, the cell obeys: it constantly recognizes and interprets environmental signals, and triggers or halts appropriate functions. However, as stated above, the cell remains unaware and ignorant of its surroundings. The third rule states that cell-environment interactions and communication are subject to binary logic: functions are on or off depending on environmental signals and internal processing.

The first two rules imply that the environment controls cellular development in an absolute manner. In other words, the 'autonomous' cell cannot make a decision on its own-its actions are totally determined by its environment. The cell 'believes' that it is actively guiding its own development, but it is only reacting to environmental conditions and is therefore guided by an external driving force. In this context, I would like to point out that the title of this article does not contain a spelling error. To express this paradox in the behaviour of a binary cell and its surroundings, I deliberately chose the new verb 'to guidle'. Cell 'guidling' thus refers to the paradox in the actions and reactions of binary cells.

A sensible scientist may conclude that these basic rules are ambiguous and might invert them, reflecting the observation that many organisms are able to change their surroundings. Humans, for example, have manipulated their environment on an unprecedented scale. Indeed, it seems that the environment itself reacts to changes in the cell and its intrinsic genics, which are themselves reactions to environmental conditions. Who then is in charge, the cell or its surroundings? This also raises the question whether a 'binary individual' can ever bypass or gain control over this cell-environment hierarchy. The only escape seems to be 'becoming aware' of the surroundings and then identifying how they restrict individual genics. If an entity succeeds in identifying the restriction mechanisms, it may be able to bypass the hierarchy or even reverse it and therefore seize power.

pplying binary logic to living enti-
ties might have real-life impli-
cations. The example I choose is cancer-cancer research and cancer therapy. It seems that, in the understanding of the first two rules, cancer cells have seized power over their environment, in this case the organism in which they evolved. They bypass regulatory mechanisms that would normally restrict their growth and proliferation, and they acquire the ability to evade the immune system, which would normally identify and destroy any aberrant cells. Furthermore, growing tumours are able to instruct the surrounding tissues to produce new blood vessels to supply them with oxygen and nutrients. It seems that this seizure of power may be the consequence of an accumulation of carcinogenic mutations in the DNA of the cell.

Humans have repeatedly declared a 'war on cancer' (von Eschenbach, 2003), but the cancer cells apparently dictate the terms under which this war is fought, an embarrassing situation that does not make any sense in terms of human logic. Would we-intelligent human beingsallow cancer cells to dictate how we fight them, seizing power not only of the patient's body but of scientific research as well? Obviously, we need a new approach to tackle cancer. Although a binary cancer cell is seemingly unaware 


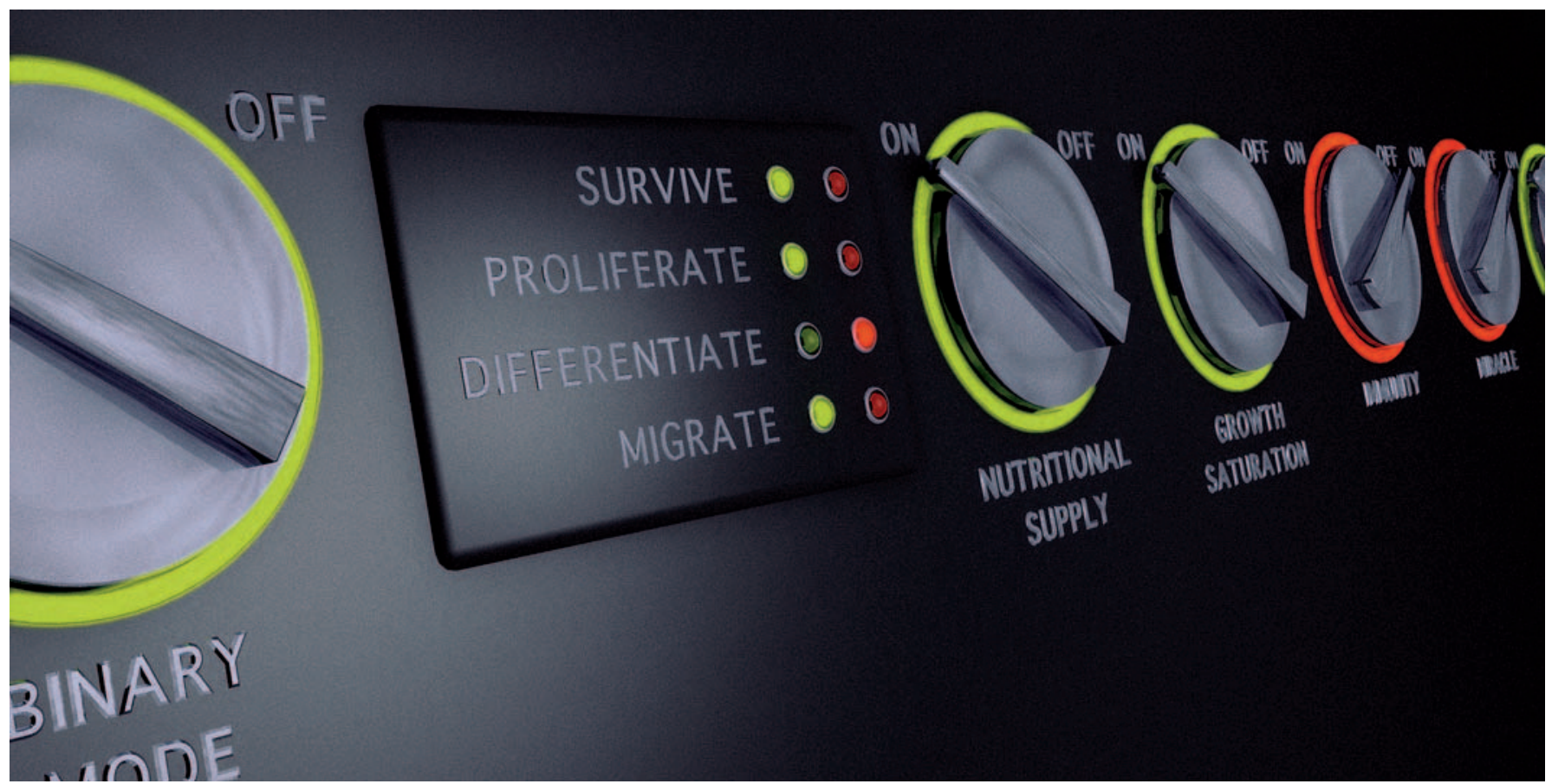

of the restrictions imposed on it by the environment, carcinogenic mutations could force the cell to unknowingly ignore one or more usual rules, even if this has devastating consequences for the whole organism-comparable with a soccer player scoring an own-goal. If this is true, should we not be able to design a smarter way to control and restrict cancer by applying binary logic?

\section{... it seems that... cancer cells have seized power over their environment, in this case the organism in which they evolved}

The spontaneous reaction of many scientists to this assumption might be, "Are you kidding me? Let's not waste time with such an oversimplification, as we know that cells, and even more multicellular organisms, are too complex to follow such simple rules. Binary logic means that something can be on or off, and there is nothing in between." Indeed, humans find it difficult to apply this to living organisms, simply because we are not accustomed to reasoning in binary terms. The more intelligent and knowledgeable we are, the more we prefer compromises and answers on a continuous scale, and the more we dislike simplifications. Therefore, a closer look at cells might reveal experimental evidence for binary logic.

argue that the binary logic of living cells is apparent in protein biosynthesis and signal transduction networks, which involve simple GTP-binding proteins. The logistics of protein biosynthesis, controlled by the elongation factors EF-Tu and EF-G, and also reflected in the actions of initiation factor IF-2 and release factor RF-2, are in fact a binary network- a circuit of on/off switches (Parmeggiani et al, 1987; Parmeggiani \& Swart, 1985; Clark et al, 1999). At the same time that this mechanism was explained, scientists also discovered various functions of the ras oncogene and the significance of GTP-binding proteins-characterized by the presence of a G-domain-as simple on/off or yes/no switches in cell signalling networks and macromolecular biosynthesis (Bourne et al, 1990, 1991). Another example are the logistics of the cell cycle, which is controlled by a few factors that act similarly to binary switches. Even the central dogma of molecular biology might just be one large 'guidling' message of how scientists should translate the genetic code into simple binary arguments: on/off, right/wrong, forwards/backwards, go/stop, and so forth. It is then not too bold to postulate that the same principle will be true for promoter action, when we have understood all events and transcription factors, which act not only on a single gene, but also on the whole genome. And what about other complex cellular mechanisms, such as intercellular communication, cell differentiation and tissue morphogenesis? I come to the conjecture that binary logic is an integral part of the life of the cell and its decision-making processes (Popper, 1962). Of course, this is a research hypothesis, which needs to be addressed and proven-or refuted-by experimental evidence.

Postulating cell behaviour on the basis of binary logic presumes the existence of mathematical equations that explain basal cell functions-cell genics-that can be observed in experiments. At first glance, this is not obvious, so I would like to borrow from other sciences that have made similar attempts to model complex systems. O ne example is psychology, which aims to understand and explain human personality types. Psychological models facilitate the classification of different individuals and therefore improve problem management in complex social interactions. One such strategy to model human personality types is the so-called

\section{The logistics of protein biosynthesis... arein fact a binary network - a circuit of on/off switches}


Myers-Briggs index (Hirsh \& Kummerow, 1989). It uses four basal parameters that can independently switch between two states, and therefore yields 16 basic types. The challenge then is to design a similar switchboard to reflect the basal parameters of cells and, ideally, to express those parameters as mathematical functions of the genes. Fortunately, our binary cell is an autonomous individual, so we can ignore all environmental influences for a while.

What are the basal functions of cells? In my view, cell behaviour may be defined by at least four simple parameters that reflect the cell's potential to survive (S), to proliferate $(P)$, to differentiate $(D)$ and to migrate $(M)$, thus defined as the SPDM index of binary cells. In any developmental stage of a cell, these four parameters can be on (1) or off (0). The binary interpretation of SPM genics is quite obvious, but $D$ genics requires an extra normalization to make it binary. [ $D=1$ ] means that the cell is not predestined for its final function; [ $D=0$ ] means that the cell is differentiating or fully differentiated. Although PDM genics are in principle reversible, $\mathrm{S}$ switching from 1 to 0 is an irreversible event-when [ $S=0$ ], the cell dies. Would it be possible to link these four basal parameters to the underlying genes? The answer might be yes, if we succeed in expressing the SPDM parameters as normalized, statistical functions of all individual genes. This might provide a link to real-life observations and an abstract connection with genomic data sets.

The next step is to introduce environmental parameters that influence the cell's SPDM index on the basis of the second rule, which states that the environment controls the actions of the cell. Again, we need mathematical operators that allow us to express (micro)environmental influences that act on the SPDM index. To this end, I return to the field of cancer research, as cancer cells are seemingly able to ignore signals from their environment: the organism. So far, the most promising and effective approaches in cancer therapy aim to strengthen the immune system and prevent angiogenesis around growing tumours (Hanahan \& W einberg, 2000). The task now is to translate these complex strategies into simple binary arguments. An immunity operator can be defined as I. When [I = 1], the immune system does not restrict the genics of the cancer cell; when [I=0], the immune system is able to identify and attack the cancer cell. By definition, [I =0] switches $S$ from 1 to 0 , thus killing the cell. The role of the immune system in relation to cancer cells would therefore be defined as $[S=I$ ] or [S = NOTI], depending on whether the cell has acquired mutations that allow it to escape the attention of the immune system.

\section{...binary logic is an integral part of the life of the cell and its decision-making processes}

A second operator would describe nutritional supply, $\mathrm{N}$, which is apparently linked to blood supply and therefore tumour-induced angiogenesis. When [ $\mathrm{N}=1]$, nutritional supply does not restrict cell genics; when $[N=0]$, nutritional supply is a limiting factor. I postulate that $[\mathrm{N}=0]$ switches $P$ from 1 to 0 , and will switch $S$ from 1 to 0 if $[N=0]$ becomes a chronic condition: $[\mathrm{N}=\mathrm{P}]$ and therefore $[\mathrm{N}=\mathrm{S}$ ] over a longer period.

A third arbitrary operator may be growth saturation, G. When [ $=1$ ], there are no restrictions on cell growth; when [G $=0$ ], environmental conditions restrict cell growth, which switches $P$ from 1 to 0 , and the cell stops proliferating. Similarly, we could define operators that switch $M$ from 0 to 1 when cancer cells migrate to cause metastatic growth throughout the body. This is a simple example, but the logic game is already becoming exciting. Taking it further, we could define even more binary operators and therefore devise binary explanations for all kinds of environmental effects on cells.

T o tackle cancer more efficiently, we should try to understand the binary logic of cell genics. If we are dealing with cancer cells that have partially and unknowingly seized power over their environment, a promising therapeutic strategy would be to push those buttons on the environmental control panel that are still functional, rather than using radio- and chemotherapy, which hit healthy cells as well. The appeal-and challenge-of practicing this cell-guidling game is that it might unveil the nature of a cancer cell without any apparent need for a priori knowledge of the genes involved, and may bypass much of the complexity.
It might be that the intense interest in understanding individual genes in cancer pathogenesis is overlooking a binary control panel that could enable researchers and clinicians to cope with the disease in a more logical and systematic way, rather than fighting a war dictated by the opponent, or targeting extremely complex defects in cellular metabolism. I believe that the complexity of the genome has so far favoured a human bias against binary logic, because it seems to be too simple an explanation compared with the complexity of living systems.

We know about the rare cases of terminal cancer patients who suddenly and miraculously improve, which are inexplicable in terms of human logic. As a scientist, I refuse to accept miracle cures and I cannot tolerate a lack of logical explanation. Could binary logic help to explain these medical miracles? These puzzling, clinical observations may hint at the existence of as yet unidentified environmental operators that apparently act on $\mathrm{S}$ and switch it from 1 to 0 . As a consequence, the binary cancer cell is doomed and the tumour disappears. Although this still looks like too simple an explanation and is not really satisfactory in terms of human logic, these medical miracles provide some evidence and support the conclusion that it may be worth looking for such binary philosopher's stones in cancer research.

$\mathrm{T}$ he first three words of the book of Genesis, "In the beginning....", raise one of the most important questions about how life on earth came about-a question that has kept theologians, philosophers and biologists busy for centuries. However, apart from the academic interest in what constitutes the first unit of life, it is also a valid question in terms of binary logic (Hofstadter, 1979), as it might help us to draw meaningful insights for more practical applications. We have gathered enormous knowledge about genomes, genes and metabolic networks, and we are developing increasingly sophisticated technologies in bioinformatics and systems biology to analyse whole genomes and organisms, but we are still

\section{I believethat the complexity of thegenome has so far favoured a human bias against binary logic...}




\section{... it counteracts our sense of logic if we compare two higher organisms without a better understanding of the origins of each}

desperately struggling and scrambling to make any sense of this gigantic pool of biological information. One common strategy is to compare our experimental system with a well-characterized model system, or to compare the responses from at least two specific cellular states or tissues. The information we gain from such comparisons consists mainly of the differences between those organisms, tissues or cellular states, rather than their common features. But it counteracts our sense of logic if we compare two higher organisms without a better understanding of the origins of each. Would it not be easier to define an original unit, however primitive, that would allow us to measure and analyse gene and metabolic activity? If the life sciences agree, it might be sensible to define a simple binary cell as a universal origin and to discuss how such a primitive 'unit of life' would look. This in turn could become the origin with which we compare higher cells and organisms to understand how complexity evolved.
I imagine that such a proposal will provoke a battery of questions and comments. How ever, new research directions, in particular systems biology and synthetic biology, already follow this route. Systems biology seeks to model the complex interplay of genes and metabolism in a cell, which, if taken to a higher order, could become the basis for the design of a primitive binary cell. Synthetic biology approaches the problem from the other side, and applies engineering principles, ultimately derived from computer science and therefore binary logic, to design genetic circuits and metabolic pathways. It will therefore be interesting to see if these ideas find their way into biological thinking and, ultimately, into biomedical research.

\section{REFERENCES}

Bourne HR, Sanders DA, McCormick F (1990) The GTPase superfamily: a conserved switch for diverse cell functions. Nature 348: 125-132 Bourne HR, Sanders DA, McCormick F (1991) The GTPase superfamily: conserved structure and molecular mechanism. Nature 349: 117-127

Clark BF, Thirup S, Kjeldgaard M , N yborg J (1999) Structural information for explaining the molecular mechanism of protein biosynthesis. FEBS Lett 452: 41-46

Hanahan D, W einberg RA (2000) The hallmarks of cancer. Cell 100: 57-70
Hirsh SK, Kummerow J (1989) UFETypes. N ew York, NY, U SA: Warner Books

H ofstadter DR (1979) Gödel, Escher, Bach: an Eternal Golden Braid. N ew York, NY, USA: Basic Books

Parmeggiani A, Swart GW (1985) Mechanism of action of kirromycin-like antibiotics. Annu Rev Microbiol 39: 557-577

Parmeggiani A, Swart GW, M ortensen KK, Jensen M , Clark BF, Dente L, Cortese R (1987) Properties of a genetically engineered $G$ domain of elongation factorTu. Proc Natl Acad Sci USA 84: 3141-3145

Popper KR (1962) Conjectures and Refutations: The Growth of Scientific Knowledge. N ew York, NY, USA: Basic Books

von Eschenbach AC (2003) N Cl sets goal of eliminating suffering and death due to cancer by 2015. J Natl Med Assoc 95: 637-639



Guido W.M. Swart is at Radboud University, Nijmegen in the Netherlands. E-mail: g.swart@ncmls.ru.nl

doi:10.1038/sj.embor.7400666 